\title{
A self-pairing theorem for tangle Floer homology
}

\author{
INA PETKOVA \\ VERA VÉRTESI
}

\begin{abstract}
We show that for a tangle $T$ with $-\partial^{0} T \cong \partial^{1} T$ the Hochschild homology of the tangle Floer homology $\widetilde{\mathrm{CT}}(T)$ is equivalent to the link Floer homology of the closure $T^{\prime}=T /\left(-\partial^{0} T \sim \partial^{1} T\right)$ of the tangle, linked with the tangle axis. In addition, we show that the action of the braid group on tangle Floer homology is faithful.
\end{abstract}

$57 \mathrm{M} 27,57 \mathrm{R} 58$

\section{Introduction}

Tangle Floer homology is an invariant of tangles in 3-manifolds with boundary $S^{2}$ or $S^{2} \sqcup S^{2}$, or in closed 3-manifolds, which takes the form of a differential graded module, bimodule, or a chain complex, respectively; see Petkova and Vértesi [9]. It behaves well under gluing and recovers knot Floer homology. Before we state the main results, we recall some definitions from [9] and make some new ones.

Definition 1.1 An $n$-marked sphere $\mathcal{S}$ is a sphere $S^{2}$ with $n$ oriented points $t_{1}, \ldots, t_{n}$ on its equator $S^{1} \subset S^{2}$ numbered respecting the orientation of $S^{1}$.

Definition 1.2 A marked $(m, n)$-tangle $\mathcal{T}$ in an oriented 3-manifold $Y$ with two boundary components $\partial^{0} Y \cong S^{2}$ and $\partial^{1} Y \cong S^{2}$ is a properly embedded $1-$ manifold $T$ with $\left(-\partial^{0} Y,-\left(\partial^{0} Y \cap \partial T\right)\right)$ identified with an $m$-marked sphere and $\left(\partial^{1} Y, \partial^{1} Y \cap \partial T\right)$ identified with an $n$-marked sphere (via orientation-preserving diffeomorphisms). We denote $-\left(\partial^{0} Y \cap \partial T\right)$ and $\partial^{1} Y \cap \partial T$ along with the ordering information by $-\partial^{0} \mathcal{T}$ and $\partial^{1} \mathcal{T}$.

Definition 1.3 A strongly marked $(m, n)$-tangle $(Y, \mathcal{T}, \gamma)$ is a marked $(m, n)$-tangle $(Y, \mathcal{T})$, along with a framed $\operatorname{arc} \gamma$ connecting $\partial^{0} Y$ to $\partial^{1} Y$ in the complement of $\mathcal{T}$ such that $\gamma$ and its framing $\lambda_{\gamma}$ (viewed as a push-off of $\gamma$ ) have ends on the equators of the two marked spheres, and we see $-\partial^{0} \mathcal{T},-\partial^{0} \gamma,-\partial^{0} \lambda_{\gamma}$ and $\partial^{1} \mathcal{T}, \partial^{1} \gamma, \partial^{1} \lambda_{\gamma}$ in this order along each equator. See Figure 1. 

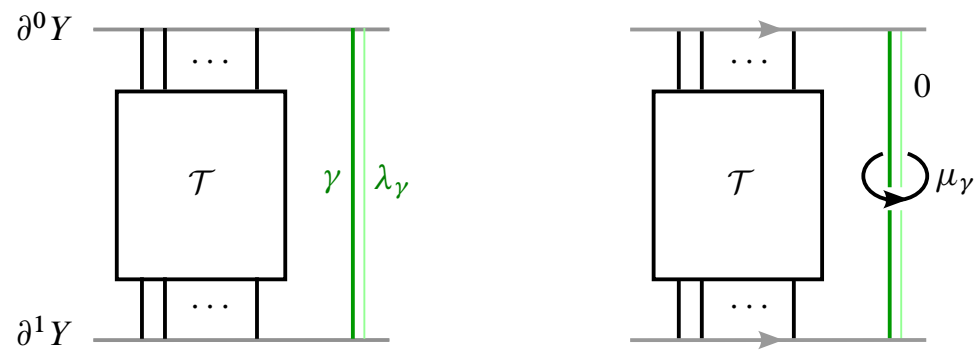

Figure 1: Left: a strongly marked tangle $(Y, \mathcal{T}, \gamma)$. Right: the surgered closure $\left(Y_{0}, T_{0}\right)$ of the tangle $(Y, \mathcal{T}, \gamma)$.

As a special case, an $(m, n)$-tangle in $\mathbb{R}^{2} \times I$ is a cobordism (contained in $[1, \infty) \times$ $\mathbb{R} \times I$ ) from $\{1, \ldots, m\} \times\{0\} \times\{0\}$ to $\{1, \ldots, n\} \times\{0\} \times\{1\}$. A tangle in $\mathbb{R}^{2} \times I$ can be thought of as a strongly marked tangle, by compactifying $\mathbb{R}^{2} \times I$ to $S^{2} \times I$, taking the images of $\mathbb{R} \times\{0\} \times\{0\}$ and $\mathbb{R} \times\{0\} \times\{1\}$ to be the equators of the marked spheres, and setting $\left(\gamma, \lambda_{\gamma}\right):=(\{(-1,0)\} \times I,\{(0,0)\} \times I)$.

We turn our attention to strongly marked tangles $(Y, \mathcal{T}, \gamma)$ with $-\partial^{0} \mathcal{T} \cong \partial^{1} \mathcal{T}$.

Definition 1.4 A strongly marked tangle $(Y, \mathcal{T}, \gamma)$ is called closable if $-\partial^{0} \mathcal{T} \cong \partial^{1} \mathcal{T}$. Given a closable tangle $(Y, \mathcal{T}, \gamma)$, we can glue it to itself to form its closure $\left(Y^{\prime}, T^{\prime}, \gamma^{\prime}\right)$ by identifying the two boundary components of $Y,-\partial^{0} Y$ and $\partial^{1} Y$, with the same marked sphere. The surgered closure of $(Y, \mathcal{T}, \gamma)$ is the pair $\left(Y_{0}, T_{0}\right)$, where the link $T_{0}$ is the union of $T^{\prime}$ and the negatively oriented meridian $\mu_{\gamma}$ of $\gamma$ in the 0 -surgery $Y_{0}=Y_{0}^{\prime}\left(\gamma^{\prime}\right)$ of $Y^{\prime}$ along the framed knot $\gamma^{\prime}$. We call $\mu_{\gamma}$ the tangle axis of the tangle $\mathcal{T}$. See Figure 1 .

When $Y$ is $S^{2} \times I$ and $\left(\gamma, \lambda_{\gamma}\right)$ is a product as above, then $Y_{0} \cong S^{3}$ and $T_{0}$ is the link formed by the closure of $T \subset \mathbb{R}^{2} \times I \subset S^{3}$ and an unknot that is the boundary of a disk containing $-\partial^{0} T \sim \partial^{1} T$, see Figure 2 . For example, for a braid $B \in \mathbb{R}^{2} \times I$, the tangle axis is precisely the braid axis.

Main Theorem Let $(Y, \mathcal{T}, \gamma)$ be a closable strongly marked tangle. Then there is an equivalence

$$
\operatorname{HH}(\widetilde{\mathrm{CT}}(Y, \mathcal{T}, \gamma)) \cong \widetilde{\mathrm{HFK}}\left(Y_{0}, T_{0}\right)
$$

Here and throughout the paper, $\widetilde{\mathrm{CT}}$ stands for the $D A$ bimodule $\widetilde{C F D T A}$ from $[9$, Section 10.3], and $\widetilde{\mathrm{HFK}}$ is the multipointed version of knot Floer homology; see for example [5; 4]. Note that both $\widetilde{C T}$ and $\widetilde{\mathrm{HFK}}$ depend on the number of basepoints in a Heegaard diagram, and the precise formulation of the Main Theorem follows in Section 3, in the form of Theorems 3.1 and 3.2. 

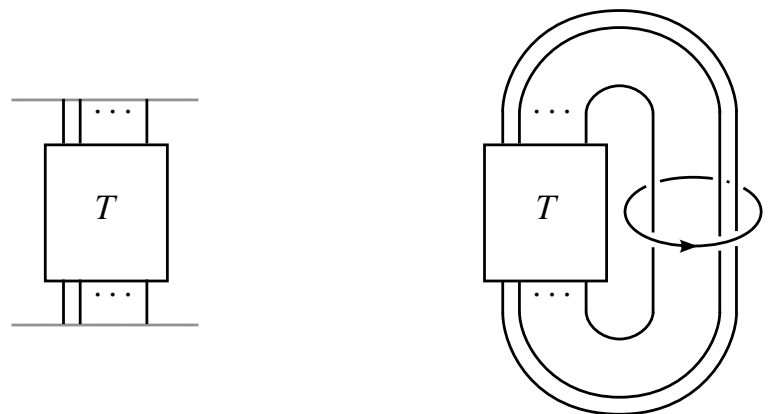

Figure 2: Left: a tangle $T \subset \mathbb{R}^{2} \times I$. Right: the corresponding link $T_{0} \subset S^{3}$.

Combined with a result of Baldwin and Grigsby [1], we get the following corollary.

Corollary Tangle Floer homology restricts to a faithful linear-categorical action of the braid group.

Acknowledgements We thank Robert Lipshitz for a helpful conversation, the referee for suggesting the Corollary to Main Theorem, and Mohammed Abouzaid and Ailsa Keating for a useful discussion of $A_{\infty}$-categories. Petkova received support from an AMS-Simons travel grant. Vértesi was supported by ERC Geodycon, OTKA grant number NK81203 and NSF grant number 1104690.

\section{Algebra review}

Let $A$ be a unital differential graded algebra over a ground ring $\boldsymbol{k}$, where $\boldsymbol{k}$ is a direct sum of copies of $\mathbb{F}_{2}=\mathbb{Z} / 2 \mathbb{Z}$. The unit gives a preferred map $\iota: \boldsymbol{k} \rightarrow A$. We assume that $A$ is augmented, ie there is a map $\epsilon: A \rightarrow \boldsymbol{k}$ such that $\epsilon(1)=1, \epsilon(a b)=\epsilon(a) \epsilon(b)$, and $\epsilon(\partial a)=0$. The augmentation ideal $\operatorname{ker} \epsilon$ is denoted by $A_{+}$.

A type DA bimodule over $(A, A)$ is a graded $\boldsymbol{k}$-bimodule $N$, together with degree 0 $\boldsymbol{k}$-linear maps

$$
\delta_{1+j}^{1}: N \otimes A[1]^{\otimes j} \rightarrow A \otimes N[1],
$$

satisfying a certain compatibility condition; see [3, Definition 2.2.42].

A $D A$ bimodule is bounded if the structure maps behave in a certain nice way; see [3, Definition 2.2.45]. We will not recall the complete definition of boundedness here, but we point out that the structures arising from nice Heegaard diagrams are bounded, and moreover the only nonzero structure maps in that case are $\delta_{1}^{1}$ and $\delta_{2}^{1}$. We will call a $D A$ bimodule nice if it is bounded and $\delta_{i}^{1}=0$ for all $i>2$. 
Given a bounded type $D A$ bimodule $N$ over $(A, A)$, one can define a chain complex $\left(N^{\circ}, \widetilde{\partial}\right)$ whose homology agrees with the Hochschild homology of the $\mathcal{A}_{\infty}$-bimodule $A \otimes N$ corresponding to $N$; see [3, Section 2.3.5]. The vector space $N^{\circ}$, called the cyclicization of $N$, is the quotient $N /[N, \boldsymbol{k}]$, where $[N, \boldsymbol{k}]$ is the submodule of $N$ generated by elements $x k-k x$, for $x \in N$ and $k \in \boldsymbol{k}$. The differential $\tilde{\partial}$ is easy to describe when $N$ is nice. We recall the construction in this special case below.

Define a cyclic rotation map $R:(A \otimes N)^{\circ} \rightarrow\left(N \otimes A_{+}\right)^{\circ}$ by

$$
R(a \otimes x)=x \otimes[(\mathrm{id}-\iota \circ \epsilon)(a)] .
$$

The map $\epsilon \otimes$ id: $A \otimes N \rightarrow \boldsymbol{k} \otimes N=N$ descends to a map $(A \otimes N)^{\circ} \rightarrow N^{\circ}$, which we will also denote $\epsilon$. We denote the cyclicizations of

$$
\delta_{1}^{1}: N \rightarrow A \otimes N \quad \text { and } \quad \delta_{2}^{1}: N \otimes A_{+} \rightarrow A \otimes N
$$

by $\delta_{1}^{1}$ and $\delta_{2}^{1}$ as well. Finally, $\tilde{\partial}$ is defined as

$$
\widetilde{\partial}=\epsilon \circ \delta_{1}^{1}+\epsilon \circ \delta_{2}^{1} \circ R \circ \delta_{1}^{1} .
$$

Given a tangle $(Y, \mathcal{T}, \gamma)$, one can represent it by a multipointed bordered Heegaard diagram $\mathcal{H}$ with two boundary components $\partial^{0} \mathcal{H}$ and $\partial^{1} \mathcal{H}$; see [9, Section 8.2]. To $-\partial^{0} \mathcal{H}$ and $\partial^{1} \mathcal{H}$ one associates differential algebras $\mathcal{A}\left(-\partial^{0} \mathcal{T}\right)$ and $\mathcal{A}\left(\partial^{1} \mathcal{T}\right)$, and to $\mathcal{H}$ a $D A$ bimodule $\widetilde{\mathrm{CT}}(\mathcal{H})$ over $\mathcal{A}\left(-\partial^{0} \mathcal{T}\right)$ and $\mathcal{A}\left(\partial^{1} \mathcal{T}\right)$. The structure maps on the bimodule are obtained by counting certain holomorphic curves in $\mathcal{H} \times I \times \mathbb{R}$; see [9, Sections 7.2 and 10.3]. For a tangle in $\mathbb{R}^{2} \times I$, the bimodule can also be defined in terms of sequences of strand diagrams corresponding to a decomposition of the tangle into elementary pieces; see [9, Sections 3 and 5.2]. We do not recall the two constructions here, but refer the reader to [9].

\section{Proofs of the main results}

We prove two versions of the Main Theorem, both via nice diagrams: the first, Theorem 3.1, deals with the general case (ungraded); the second, Theorem 3.2, deals with the case when the tangle is in $\mathbb{R}^{2} \times I$.

Tangle Floer homology is an invariant of the tangle: if $\mathcal{H}_{1}$ and $\mathcal{H}_{2}$ are Heegaard diagrams for $(Y, \mathcal{T}, \gamma)$ with $2 k_{1}$ and $2 k_{2}$ basepoints, respectively, and $k_{1} \geq k_{2}$, then

$$
\widetilde{\mathrm{CT}}\left(\mathcal{H}_{1}\right) \simeq \widetilde{\mathrm{CT}}\left(\mathcal{H}_{2}\right) \otimes\left(\mathbb{F}_{2} \oplus \mathbb{F}_{2}\right)^{\otimes\left(k_{1}-k_{2}\right)} .
$$

For a closable tangle $-\partial^{0} \mathcal{T}$ is isomorphic to $\partial^{1} \mathcal{T}$, so the algebras $\mathcal{A}\left(-\partial^{0} \mathcal{T}\right)$ and $\mathcal{A}\left(\partial^{1} \mathcal{T}\right)$ are the same, and one can take the Hochschild homology of the bimodule. 


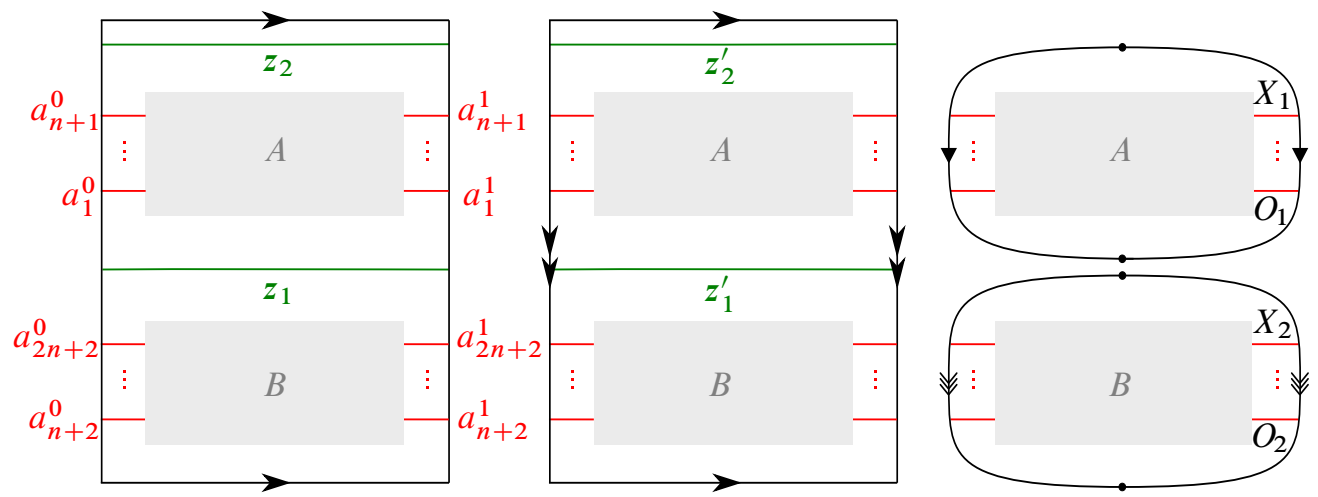

Figure 3: Left: a Heegaard diagram $\mathcal{H}$ for a closable tangle $(Y, \mathcal{T}, \gamma)$. The left edge is $\partial^{0} \mathcal{H}$ and the right edge is $\partial^{1} \mathcal{H}$. Middle: the diagram $\mathcal{H}^{\prime}$ obtained by self-gluing $\mathcal{H}$. Right: the Heegaard diagram $\mathcal{H}^{\circ}$ obtained by surgery on the two green curves $z_{1}^{\prime}$ and $z_{2}^{\prime}$ on $\mathcal{H}^{\prime}$.

Theorem 3.1 If $\mathcal{H}$ is Heegaard diagram with $2 k$ basepoints for a closable strongly marked tangle $(Y, \mathcal{T}, \gamma)$, and $\mathcal{H}^{\circ}$ is a diagram with $2 k+4$ basepoints for $\left(Y_{0}, T_{0}\right)$, for some $k$, then

$$
\mathrm{HH}(\widetilde{\mathrm{CT}}(\mathcal{H})) \cong \widetilde{\mathrm{HFK}}\left(\mathcal{H}^{\circ}\right)
$$

Note that in [9], gradings for tangle Floer homology are only defined when the underlying manifold is $S^{2} \times I$ or $B^{3}$, so Theorem 3.1 only claims an ungraded isomorphism.

Proof The proof is very similar to that of [3, Theorem 14]. By invariance under Heegaard moves, it suffices to prove the theorem for one choice of $\mathcal{H}$, and one choice of $\mathcal{H}^{\circ}$ with 4 more basepoints than $\mathcal{H}$.

Let $(Y, \mathcal{T}, \gamma)$ be a closable strongly marked $(n, n)$-tangle, and $\mathcal{H}=(\Sigma, \boldsymbol{\alpha}, \boldsymbol{\beta}, \mathbb{X}, \mathbb{O}, \boldsymbol{z})$ be a nice bordered Heegaard diagram for $(Y, \mathcal{T}, \gamma)$, as in [9, Proposition 12.1]. Glue $\mathcal{H}$ to itself by identifying $-\partial^{0} \mathcal{H}$ and $\partial^{1} \mathcal{H}$, and call the result $\mathcal{H}^{\prime}$ (note that this is not a valid Heegaard diagram). Recall that $z=\left\{z_{1}, z_{2}\right\}$ is a set of two arcs in $\Sigma \backslash(\boldsymbol{\alpha} \cup \boldsymbol{\beta})$ with boundary on $\partial \Sigma \backslash \boldsymbol{\alpha}$, oriented from the left to the right boundary, and let $\boldsymbol{z}_{1}^{\prime}$ and $z_{2}^{\prime}$ be the resulting closed curves in $\mathcal{H}^{\prime}$. Surger $\mathcal{H}^{\prime}$ along $z_{1}^{\prime}$ and $z_{2}^{\prime}$, and place 4 basepoints in the 4 resulting regions: $X_{1}, O_{1}, X_{2}$, and $O_{2}$ in the region whose boundary contains $a_{n+1}^{0}, a_{1}^{0}, a_{2 n+2}^{0}$, and $a_{n+2}^{0}$, respectively. The result is a diagram $\mathcal{H}^{\circ}=\left(\Sigma^{\circ}, \boldsymbol{\alpha}^{\circ}, \boldsymbol{\beta}^{\circ}, \mathbb{X}^{\circ}, \mathbb{O}^{\circ}\right)$; see Figure 3.

Recall that originally $(Y, \mathcal{T})$ was obtained by attaching a two handle to the pair $\left(Y_{\mathrm{dr}}, \mathcal{T}_{\mathrm{dr}}\right)$ that we got from a drilled diagram on $\Sigma-N\left(z_{2}\right)$ by the usual handle attachments. Thus, the Heegaard diagram $\mathcal{H}^{\prime \prime}$ that we get by only doing surgery on $\mathcal{H}^{\prime}$ along $z_{2}^{\prime}$ 
describes $\left(Y^{\prime}-N\left(\gamma^{\prime}\right), T^{\prime}\right)$, with $z_{1}^{\prime}$ lying on the boundary of $N\left(\gamma^{\prime}\right)$. Now the surgery along $z_{1}^{\prime}$ on $\mathcal{H}^{\prime \prime}$ simply results in the Dehn filling of $\left(Y^{\prime}-N\left(\gamma^{\prime}\right), T^{\prime}\right)$ with framing $z_{1}^{\prime}$. The knot described by $X_{1}, O_{1}, X_{2}$, and $O_{2}$ goes through the former $z_{2}$ and $z_{1}$ on the Heegaard diagram, thus indeed describes a meridian for $T^{\prime}$. This means that $\mathcal{H}^{\circ}$ is a Heegaard diagram for $\left(Y_{0}, T_{0}\right)$.

Observe that the generators of $\mathcal{H}^{\prime}$, or equivalently the generators of $\mathcal{H}^{\circ}$, correspond to generators $\boldsymbol{x}$ of $\mathcal{H}$ with $\bar{o}^{0}(\boldsymbol{x})=o^{1}(\boldsymbol{x})$, whereas in [9] $o^{1}(\boldsymbol{x})$ (respectively $\bar{o}^{0}(\boldsymbol{x})$ ) denotes the set of $\alpha$ arcs that are occupied (not occupied) by $x$ on $\alpha^{1}$ (and $\alpha^{0}$ ).

Denote the algebra $\mathcal{A}\left(\partial^{1} \mathcal{T}\right) \cong \mathcal{A}\left(-\partial^{0} \mathcal{T}\right)$ by $\mathcal{A}$, and its ring of idempotents by $\boldsymbol{k}$. Recall that $\mathcal{A}$ has a basis over $\mathbb{F}_{2}$ consisting of strand diagrams [9, Section 7]. We define the augmentation map $\epsilon: \mathcal{A} \rightarrow \boldsymbol{k}$ on this basis explicitly: it is the identity on generators in $\boldsymbol{k} \subset \mathcal{A}$ and zero on generators $a \notin \boldsymbol{k} \subset \mathcal{A}$. The structure maps on the $D A$ bimodule $\widetilde{\mathrm{CT}}(\mathcal{H})$ count the following types of domains; see [9, Sections 10 and 12]:

(1) Empty provincial rectangles and bigons. These contribute to $\delta_{1}^{1}$, with image in $\boldsymbol{k} \otimes \widetilde{\mathrm{CT}}(\mathcal{H}) \subset \mathcal{A} \otimes \widetilde{\mathrm{CT}}(\mathcal{H})$.

(2) Empty rectangles that intersect $\partial^{0} \mathcal{H}$ (the left boundary of $\mathcal{H}$ ). These contribute to $\delta_{1}^{1}$, with image in $\mathcal{A}_{+} \otimes \widetilde{\mathrm{CT}}(\mathcal{H}) \subset \mathcal{A} \otimes \widetilde{\mathrm{CT}}(\mathcal{H})$.

(3) Sets of empty rectangles, each of which intersects $\partial^{1} \mathcal{H}$ (the right boundary of $\mathcal{H}$ ). These comprise $\delta_{2}^{1}$, whose image is entirely contained in $\boldsymbol{k} \otimes \widetilde{\mathrm{CT}}(\mathcal{H})$.

The differential on the Hochschild complex $\left(\widetilde{\mathrm{CT}}(\mathcal{H})^{\circ}, \widetilde{\partial}\right)$ then counts the following domains on $\mathcal{H}$. The map $\epsilon \circ \delta_{1}^{1}$ counts provincial rectangles and bigons, and then forgets the idempotent component of the output. These are exactly the empty rectangles and bigons in $\mathcal{H}^{\circ}$ that do not cross $-\partial^{0} \mathcal{H}=\partial^{1} \mathcal{H}$. The map $\epsilon \circ \delta_{2}^{1} \circ R \circ \delta_{1}^{1}$ counts rectangles as follows. Since the rotation map $R$ is zero on elements $e \otimes x$ with $e \in \boldsymbol{k}$, only the part of $\delta_{1}^{1}$ that counts domains of Type (2) contributes. Thus, the image of $R \circ \delta_{1}^{1}$ is generated by elements of form $y \otimes a$, where $a \in \mathcal{A}_{+}$is a generator with only one moving strand. Thus, the part of $\delta_{2}^{1}$ that contributes to $\epsilon \circ \delta_{2}^{1} \circ R \circ \delta_{1}^{1}$ counts individual empty rectangles that intersect $\partial^{1} \mathcal{H}$. To sum up, $\epsilon \circ \delta_{2}^{1} \circ R \circ \delta_{1}^{1}$ counts pairs of empty rectangles, one with an edge on $\partial^{0} \mathcal{H}$ and one with an edge on $\partial^{1} \mathcal{H}$, which glue up to a rectangle after the identification $-\partial^{0} \mathcal{H} \sim \partial^{1} \mathcal{H}$. These are exactly the empty rectangles in $\mathcal{H}^{\circ}$ that cross $-\partial^{0} \mathcal{H} \sim \partial^{1} \mathcal{H}$.

Thus, $\left(\widetilde{\mathrm{CT}}(\mathcal{H})^{\circ}, \widetilde{\partial}\right) \cong \widetilde{\mathrm{CFK}}\left(\mathcal{H}^{\circ}\right)$.

For the special case of a tangle $T$ in $\mathbb{R}^{2} \times I$, we state a graded version. In this case, $\mathrm{HH}(\widetilde{\mathrm{CT}}(\mathcal{H}))$ inherits the Maslov and Alexander gradings $M$ and $A$ from $\widetilde{\mathrm{CT}}(\mathcal{H})$, and also carries a strands grading $S$ (counting the number of occupied $\alpha$-arcs that 
touch $\left.\partial^{1} \mathcal{H}\right)$. If $\mathcal{H}_{1}$ and $\mathcal{H}_{2}$ are Heegaard diagrams for $T$ with $2 k_{1}$ and $2 k_{2}$ basepoints, respectively, and $k_{1} \geq k_{2}$, then $\widetilde{\mathrm{CT}}\left(\mathcal{H}_{1}\right) \simeq \widetilde{\mathrm{CT}}\left(\mathcal{H}_{2}\right) \otimes\left(\mathbb{F}_{2} \oplus \mathbb{F}_{2}\right)^{\otimes\left(k_{1}-k_{2}\right)}$, where each tensor factor $\mathbb{F}_{2} \oplus \mathbb{F}_{2}$ has one summand in $(M, A, S)$ trigrading $(0,0,0)$ and one in trigrading $(-1,-1,0)$.

Let $l$ be the number of components of $T_{0}$, and label the them $L_{0}=\mu_{\gamma}, L_{1}, \ldots, L_{l-1}$. The link Floer homology of the Heegaard diagram $\mathcal{H}^{\circ}$ for $T_{0}$ described earlier, $\widetilde{\mathrm{HFL}}\left(\mathcal{H}^{\circ}\right)$, is multigraded, with Maslov grading $M$ in $\mathbb{Z}+\frac{1}{2}(l-1)$ and Alexander multigrading $\left(A_{0}, \ldots, A_{l-1}\right)$ in $\left(\frac{1}{2} \mathbb{Z}\right)^{l}$, with each $\frac{1}{2} \mathbb{Z}$ factor corresponding to a component of the link [8]. Let $\widetilde{\mathrm{HFL}}\left(\mathcal{H}^{\circ}, 0\right)$ be $\widetilde{\mathrm{HFL}}\left(\mathcal{H}^{\circ}\right)$ with multigrading collapsed to a trigrading by $M, A_{0}$, and $A^{\prime}:=A_{1}+\cdots+A_{l-1}=A-A_{0}$ (here $A=A_{0}+\cdots+A_{l-1}$ is the Alexander grading on $\left.\widetilde{\mathrm{HFK}}\left(\mathcal{H}^{\circ}\right)\right)$.

Theorem 3.2 Let $\mathcal{H}$ be a Heegaard diagram with $2 k$ basepoints for a tangle $T$ in $\mathbb{R}^{2} \times I$ with $-\partial^{0} T \cong \partial^{1} T$, and let $\mathcal{H}^{\circ}$ be a diagram with $2 k+4$ basepoints for the surgered closure $T_{0}$, with 4 of the basepoints corresponding to the component $\mu_{\gamma}$. Then there is an isomorphism

$$
\mathrm{HH}(\widetilde{\mathrm{CT}}(\mathcal{H})) \cong \widetilde{\mathrm{HFL}}\left(\mathcal{H}^{\circ}, 0\right)
$$

which respects the trigrading in the following sense. If the isomorphism maps a homogeneous element $\boldsymbol{x} \in \mathrm{HH}(\widetilde{\mathrm{CT}}(\mathcal{H}))$ to an element $\boldsymbol{y} \in \widetilde{\mathrm{HFL}}\left(\mathcal{H}^{\circ}, 0\right)$, then $\boldsymbol{y}$ is homogeneous and

$$
\begin{aligned}
& M(y)=M(x)+S(x)-a-1, \\
& A^{\prime}(y)=A(x)-S(x)+\frac{1}{2} l+n-a-1, \\
& A_{0}(y)=S(x)-\frac{1}{2}(n+1),
\end{aligned}
$$

where $n=\left|\partial^{1} T\right|, a$ is the number of positively oriented points in $\partial^{1} T$, and $l$ is the number of components of $T_{0}$.

Proof Again, by invariance under Heegaard moves, it suffices to prove the theorem for any one specific choice of $\mathcal{H}$ and $\mathcal{H}^{\circ}$ with the prescribed relative number of basepoints. We already discussed the isomorphism in the proof of Theorem 3.1. It remains to identify the gradings.

Let $\mathcal{H}$ be a Heegaard diagram for $T$ obtained by plumbing annular bordered grid diagrams, as in [9, Section 4]. By gluing on a diagram for the straight strands $\partial^{1} T \times I$ if necessary, we may assume that $\mathcal{H}$ has even genus, which we denote by $2 g$ (this makes for an easier gradings argument). See the top diagram in Figure 4. We modify $\mathcal{H}$ to a diagram $\mathcal{H}^{\circ}$ for $T_{0}$, as in the proof of Theorem 3.1. See the bottom diagram in Figure 4. 

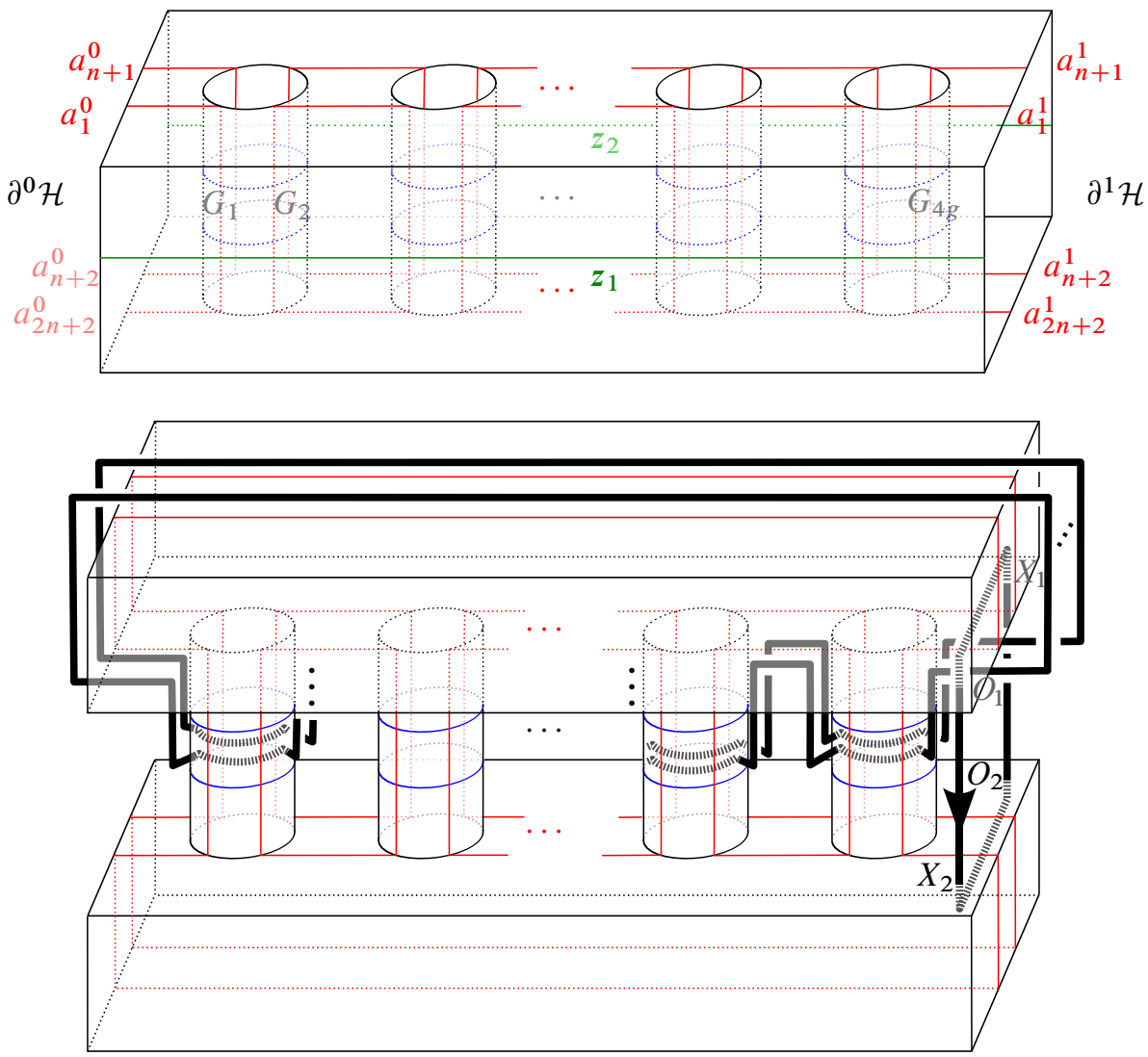

Figure 4: Top: a diagram for $T$ in $S^{2} \times I$. There are two grids on each vertical annulus, $X \mathrm{~s}$ and $O \mathrm{~s}$ omitted for simplicity. Bottom: the corresponding diagram for $T_{0}$ in $S^{3}$, along with $T_{0}$ in bold black.

Call the part of $\mathcal{H}^{\circ}$ away from the four regions resulting from the surgery on $z_{i}^{\prime}$ the nice part of $\mathcal{H}^{\circ}$ (this is the part that is the plumbing of grid diagrams). As in Figure 3, we can draw $\mathcal{H}^{\circ}$ on the plane, as the union of two $2 g$-punctured disks with certain identifications of the boundary, see Figure 7. As seen on Figure 7, we refer to the top/bottom disk as the top/bottom half of $\mathcal{H}^{\circ}$, respectively.

Denote the set of generators of $\mathcal{H}$ by $\mathfrak{S}(\mathcal{H})$, and the subset of generators with $i$ occupied $\alpha$-arcs on the right by $\mathfrak{S}_{i}(\mathcal{H})$. Denote the subsets of $\mathfrak{S}(\mathcal{H})$ and $\mathfrak{S}_{i}(\mathcal{H})$ that correspond to generators of $\mathcal{H}^{\circ}$ by $\mathfrak{S}(\mathcal{H})^{\circ}$ and $\mathfrak{S}_{i}(\mathcal{H})^{\circ}$, and the corresponding sets of generators of $\mathcal{H}^{\circ}$ by $\mathfrak{S}\left(\mathcal{H}^{\circ}\right)$ and $\mathfrak{S}_{i}\left(\mathcal{H}^{\circ}\right)$, respectively. For a generator $\boldsymbol{x} \in \mathfrak{S}(\mathcal{H})$, denote the corresponding generator in $\mathfrak{S}\left(\mathcal{H}^{\circ}\right)$ by $\boldsymbol{x}^{\circ}$. Define a strands grading on generators by $S\left(x_{i}^{\circ}\right)=i$ for $\boldsymbol{x}_{i}^{\circ} \in \mathfrak{S}_{i}\left(\mathcal{H}^{\circ}\right)$. 


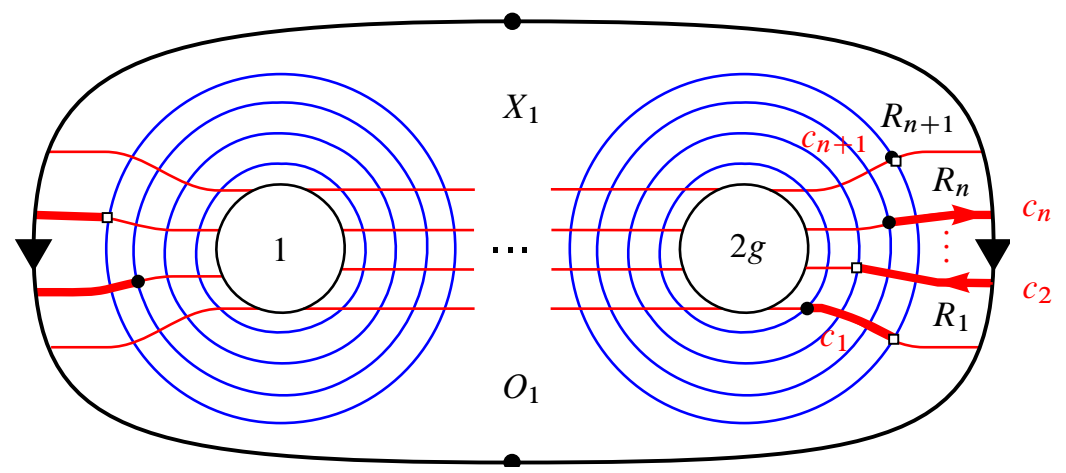

Figure 5: The top half of $\mathcal{H}^{\circ}$, along with the arcs $c_{i}$ in thick red $\left(c_{n+1}\right.$ is just a point).

Since $\mathcal{H}^{\circ}$ is a diagram for $S^{3}$, any two generators are connected by a domain. Let $\boldsymbol{x}^{\circ}, \boldsymbol{y}^{\circ}$ be elements of $\mathfrak{S}\left(\mathcal{H}^{\circ}\right)$, and $B$ an element of $\pi_{2}\left(\boldsymbol{x}^{\circ}, \boldsymbol{y}^{\circ}\right)$. By adding regions of $\Sigma^{\circ} \backslash \boldsymbol{\alpha}^{\circ}$, we can assume that the domain of $B$ is contained entirely in the top half of $\mathcal{H}^{\circ}$ and has zero multiplicity in the lowest region (the one containing $O_{1}$ ) of the top half of $\mathcal{H}^{\circ}$. The oriented boundary of $B$ splits into two pieces: $\partial^{\alpha} B \subset \boldsymbol{\alpha}^{\circ}$ and $\partial^{\beta} B \subset \boldsymbol{\beta}^{\circ}$. The piece $\partial^{\alpha} B$ is the union of arcs in $\boldsymbol{\alpha}^{\circ}$ such that $\partial\left(\partial^{\alpha} B\right)=\boldsymbol{y}^{\circ}-\boldsymbol{x}^{\circ}$.

Let $\alpha_{1}, \ldots, \alpha_{n+1}$ be the $\alpha$-circles in $\mathcal{H}^{\circ}$ resulting from the gluing of the $\alpha$-arcs in $\mathcal{H}$, labeled so that $a_{i}^{0} \in \alpha_{i}$, and let $x_{i}=\boldsymbol{x}^{\circ} \cap \alpha_{i}, y_{i}=\boldsymbol{y}^{\circ} \cap \alpha_{i}$. Below, we turn our attention to the oriented $\operatorname{arcs} c_{i}:=\partial^{\alpha} B \cap \alpha_{i}$, and to each $c_{i}$ we associate a number $t_{i} \in\{-1,0,1\}$. Since $B$ is contained in the top half of $\mathcal{H}^{\circ}$, there are three possibilities for each $c_{i}$ :

- $c_{i}$ is contained in the rightmost/leftmost grid if and only if $x_{i}$ and $y_{i}$ are; in this case, define $t_{i}=0$.

- $c_{i}$ covers both the rightmost and the leftmost grid, and is oriented to the right, as seen on Figure 5, if and only if $x_{i}$ is in the rightmost grid and $y_{i}$ is in the leftmost grid; in this case, define $t_{i}=1$.

- $c_{i}$ covers both the rightmost and the leftmost grid, and is oriented to the left, as seen on Figure 5, if and only if $x_{i}$ is in the leftmost grid and $y_{i}$ is in the rightmost grid; in this case, define $t_{i}=-1$.

For $1 \leq i \leq n$, let $R_{i}$ be the region of $\Sigma^{\circ} \backslash\left(\boldsymbol{\alpha}^{\circ} \cup \boldsymbol{\beta}^{\circ}\right)$ containing the image of the interval $\left(a_{i}^{0}, a_{i+1}^{0}\right)$ in $\Sigma^{\circ}$, and let $R_{n+1}$ be the topmost region of the top half of $\mathcal{H}^{\circ}$. See Figure 5. 
It is not hard to see that the multiplicity of $B$ at each $R_{i}$ is $t_{1}+\cdots+t_{i}$, and that the multiplicity of $B$ at $R_{n+1}$ is zero if and only if the generators $\boldsymbol{x}$ and $\boldsymbol{y}$ of $\mathcal{H}$ corresponding to $\boldsymbol{x}^{\circ}$ and $\boldsymbol{y}^{\circ}$ occupy the same number of arcs on the right.

Suppose $\boldsymbol{x}^{\circ}, \boldsymbol{y}^{\circ} \in \mathfrak{S}_{i}\left(\mathcal{H}^{\circ}\right)$. By the above, $\boldsymbol{x}^{\circ}$ and $\boldsymbol{y}^{\circ}$ are connected by a domain $B$ contained entirely in the nice part of $\mathcal{H}^{\circ}$, ie $n_{X_{1}}(B)=n_{X_{2}}(B)=n_{O_{1}}(B)=n_{O_{2}}(B)=0$, so $A_{0}(\boldsymbol{x})=A_{0}(\boldsymbol{y})$. Then $B$ is the result of self-gluing a domain $B^{\prime}$ in $\mathcal{H}$. Note that the left and right multiplicities of $B^{\prime}$ match up, ie if $p_{0} \in \partial^{0} \mathcal{H}$ and $p_{1} \in \partial^{1} \mathcal{H}$ are points that are identified in $\mathcal{H}^{\circ}$, then $m\left(\partial^{0} B^{\prime}, p_{0}\right)=-m\left(\partial^{1} B^{\prime}, p_{1}\right)$. Recall the definitions of the sets $S_{\mathbb{O}}^{i}, S_{\mathbb{X}}^{i}, S_{\boldsymbol{x}}^{i}, S_{\boldsymbol{y}}^{i}, \bar{S}_{\mathbb{O}}^{i}, \bar{S}_{\mathbb{X}}^{i}, \bar{S}_{\boldsymbol{x}}^{i}, \bar{S}_{\boldsymbol{y}}^{i}$, and of the gradings of domains from [9, Section 11.2]. For each subscript $\bullet \in\{\mathbb{O}, \mathbb{X}, \boldsymbol{x}, \boldsymbol{y}\}, p_{0} \in \bar{S}_{\bullet}^{0}$ if and only if $p_{1} \in S_{\bullet}^{1}$. Further, $e(B)=e\left(B^{\prime}\right)$, and $n_{p}(B)=n_{p}\left(B^{\prime}\right)$ for any point $p$. Then

$$
\begin{aligned}
M\left(B^{\prime}\right) & =-e\left(B^{\prime}\right)-n_{\boldsymbol{x}}\left(B^{\prime}\right)-n_{\boldsymbol{y}}\left(B^{\prime}\right)+\frac{1}{2} m\left(\left[\partial^{\partial} B^{\prime}\right], \bar{S}_{\boldsymbol{x}}^{0}+\bar{S}_{\boldsymbol{y}}^{0}+S_{\boldsymbol{x}}^{1}+S_{\boldsymbol{y}}^{1}\right) \\
& -m\left(\left[\partial^{\partial} B^{\prime}\right], \bar{S}_{O}^{0}+S_{O}^{1}\right)+2 n_{\mathbb{O}}\left(B^{\prime}\right) \\
& =-e(B)-n_{\boldsymbol{x}}(B)-n_{\boldsymbol{y}}(B)+2 n_{\mathbb{O}}(B) \\
& =M(B), \\
A\left(B^{\prime}\right) & =\frac{1}{2} m\left(\left[\partial^{\partial} B^{\prime}\right], \bar{S}_{\mathbb{X}}^{0}-\bar{S}_{O}^{0}+S_{\mathbb{X}}^{1}-S_{O}^{1}\right)+n_{\mathbb{O}}\left(B^{\prime}\right)-n_{\mathbb{X}}\left(B^{\prime}\right) \\
& =n_{\mathbb{O}}(B)-n_{\mathbb{X}}(B) \\
& =A(B) .
\end{aligned}
$$

Thus, the relative $(M, A)$ gradings are the same in $\widetilde{\mathrm{CT}}(\mathcal{H})$ as in $\widehat{\mathrm{CFK}}\left(\mathcal{H}^{\circ}\right)$, and the relative $A_{0}$ grading is zero, ie

$$
\begin{aligned}
M\left(x^{\circ}\right)-M\left(y^{\circ}\right) & =M(x)-M(y), \\
A\left(x^{\circ}\right)-A\left(y^{\circ}\right) & =A(x)-A(y), \\
A_{0}\left(x^{\circ}\right)-A_{0}\left(y^{\circ}\right) & =0 .
\end{aligned}
$$

Next, we compare the gradings of generators with distinct numbers of occupied arcs on the right. The plumbing of $4 g$ grid diagrams for $\mathcal{H}$ corresponds to a sequence of $4 g$ shadows $\mathcal{P}_{1}, \ldots, \mathcal{P}_{4 g}$; see $\left[9\right.$, Sections 3 and 4] and Figure 6. Suppose $\mathfrak{S}_{i}\left(\mathcal{H}^{\circ}\right) \neq \varnothing$ and $\mathfrak{S}_{i+j}\left(\mathcal{H}^{\circ}\right) \neq \varnothing$ for some $i, j$, and let $\boldsymbol{x}_{i} \in \mathfrak{S}_{i}(\mathcal{H})^{\circ}, \boldsymbol{x}_{i+j} \in \mathfrak{S}_{i+j}(\mathcal{H})^{\circ}$. The generator $\boldsymbol{x}_{i+j}$ has $j$ more strands than $\boldsymbol{x}_{i}$ in each even-indexed shadow $\mathcal{P}_{2 t}$. Choose one strand of $\boldsymbol{x}_{i+j}$ in each $\mathcal{P}_{2 t}$, and let $p_{2 t-1}$ and $p_{2 t}$ be the endpoints of this strand in $\partial^{0} \mathcal{P}_{2 t}$ and $\partial^{1} \mathcal{P}_{2 t}$, respectively. Replacing these $2 g$ strands with the strands from $p_{2 t}$ to $p_{2 t+1}$ produces a generator $\boldsymbol{x}_{i+j-1} \in \mathfrak{S}_{i+j-1}(\mathcal{H})^{\circ}$. Repeating this procedure shows that $\mathfrak{S}_{k}(\mathcal{H})^{\circ} \neq \varnothing$ for every $i+1 \leq k \leq i+j-1$ as well. 
Then it suffices to choose two generators $\boldsymbol{x}_{i} \in \mathfrak{S}_{i}(\mathcal{H})^{\circ}$ and $\boldsymbol{x}_{i+1} \in \mathfrak{S}_{i+1}(\mathcal{H})^{\circ}$ for each $i$ such that $\mathfrak{S}_{i}(\mathcal{H})^{\circ} \neq \varnothing$ and $\mathfrak{S}_{i+1}(\mathcal{H})^{\circ} \neq \varnothing$, and understand the relative $\left(M, A, A_{0}\right)$ grading for the corresponding generators $\boldsymbol{x}_{i}^{\circ}, \boldsymbol{x}_{i+1}^{\circ}$. Let $\boldsymbol{x}_{i+1} \in \mathfrak{S}_{i+1}(\mathcal{H})^{\circ}$ and let $p_{0}, \ldots, p_{4 g} \simeq p_{0}$ be as above. Modify $\boldsymbol{x}_{i+1}$ as follows. Let $q_{t}$ be the topmost point in $\partial^{1} \mathcal{P}_{t}$. The strand at $q_{t}$ may be contained in $\mathcal{P}_{t}$ or in $\mathcal{P}_{t+1}$. Starting at $t=1$, and moving up to $t=4 g$ (identify $\partial^{1} \mathcal{P}_{4 g}$ with $\partial^{0} \mathcal{P}_{1}$ ), do the following exchanges of strands. If $q_{t} \neq p_{t}$, take the two distinct strands with ends at $q_{t}$ and at $p_{t}$, and exchange their endpoints on $\partial^{0} \mathcal{P}_{t}$. In other words, if one strand connects $p_{t}$ to another point $p_{t}^{\prime}$, and the other strand connected $q_{t}$ to another point $q_{t}^{\prime}$, then replace the two strands with a strand connecting $p_{t}$ to $q_{t}^{\prime}$ and a strand connecting $q_{t}$ to $p_{t}^{\prime}$. In this modified $\boldsymbol{x}_{i+1}$, there is a strand connecting $q_{2 t-1}$ to $q_{2 t}$, for $1 \leq t \leq 2 g$. Let $\boldsymbol{x}_{i}$ be the generator obtained from $x_{i+1}$ by replacing these strands with strands from $q_{2 t}$ to $q_{2 t+1}$, as above. Now $\boldsymbol{x}_{i} \in \mathfrak{S}_{i}(\mathcal{H})^{\circ}$ and $\boldsymbol{x}_{i+1} \in \mathfrak{S}_{i+1}(\mathcal{H})^{\circ}$ agree almost everywhere, except that $\boldsymbol{x}_{i+1}$ contains the strand at the very top of each even-indexed shadow, and $\boldsymbol{x}_{i}$ contains the strand at the very top of each odd-indexed shadow. The Maslov and Alexander gradings on strand generators are defined by counting various intersections of strands, see [9, Section 3.4], and one sees that $M\left(\boldsymbol{x}_{i+1}\right)=M\left(\boldsymbol{x}_{i}\right)$ and $A\left(\boldsymbol{x}_{i+1}\right)=A\left(\boldsymbol{x}_{i}\right)$.

Switching back to Heegaard diagrams, $\boldsymbol{x}_{i}$ and $\boldsymbol{x}_{i+1}$ differing in the above way is equivalent to saying that the $4 g-$ gon $R_{n+1}$ connects $x_{i+1}^{\circ}$ to $x_{i}^{\circ}$. Since $e\left(R_{n+1}\right)=$ $1-g, n_{\boldsymbol{x}_{i+1}^{\circ}}\left(R_{n+1}\right)=g / 2=n_{\boldsymbol{x}_{i}^{\circ}}\left(R_{n+1}\right), n_{X_{1}}\left(R_{n+1}\right)=1$ and $n_{p}\left(R_{n+1}\right)=0$ for any other $p \in \mathbb{X} \cup \mathbb{O}$, we see that

$$
\begin{aligned}
M\left(\boldsymbol{x}_{i+1}^{\circ}\right)-M\left(\boldsymbol{x}_{i}^{\circ}\right) & =1, \\
A\left(\boldsymbol{x}_{i+1}^{\circ}\right)-A\left(\boldsymbol{x}_{i}^{\circ}\right) & =0, \\
A_{0}\left(\boldsymbol{x}_{i+1}^{\circ}\right)-A_{0}\left(\boldsymbol{x}_{i}^{\circ}\right) & =1 .
\end{aligned}
$$

So for $i<j$ and arbitrary $\boldsymbol{x}_{i}^{\circ} \in \mathfrak{S}_{i}\left(\mathcal{H}^{\circ}\right), \boldsymbol{x}_{j}^{\circ} \in \mathfrak{S}_{j}\left(\mathcal{H}^{\circ}\right)$, we have

$$
\begin{aligned}
M\left(x_{j}^{\circ}\right)-M\left(x_{i}^{\circ}\right) & =M\left(x_{j}\right)-M\left(x_{i}\right)+j-i, \\
A\left(x_{j}^{\circ}\right)-A\left(x_{i}^{\circ}\right) & =A\left(x_{j}\right)-A\left(x_{i}\right), \\
A_{0}\left(x_{j}^{\circ}\right)-A_{0}\left(x_{i}^{\circ}\right) & =j-i .
\end{aligned}
$$

The argument that the isomorphism respects the absolute gradings is analogous to the one from [9, Section 6]. With the $4 g$ grids arranged as in Figure 6, indexed $G_{1}, \ldots, G_{4 g}$ from left to right, let $\boldsymbol{x}_{\mathbb{O}}^{\circ}$ be the generator formed by the bottom left corner $x_{j}$ of each $O_{j}$ in $G_{4 i}$ and $G_{4 i+1}$, the top right corner $x_{j}$ of each $O_{j}$ in $G_{4 i+2}$ and $G_{4 i+3}$, the very top right corner $x_{4 i+1}^{\prime}$ of each grid $G_{4 i+1}$, and the bottom left 

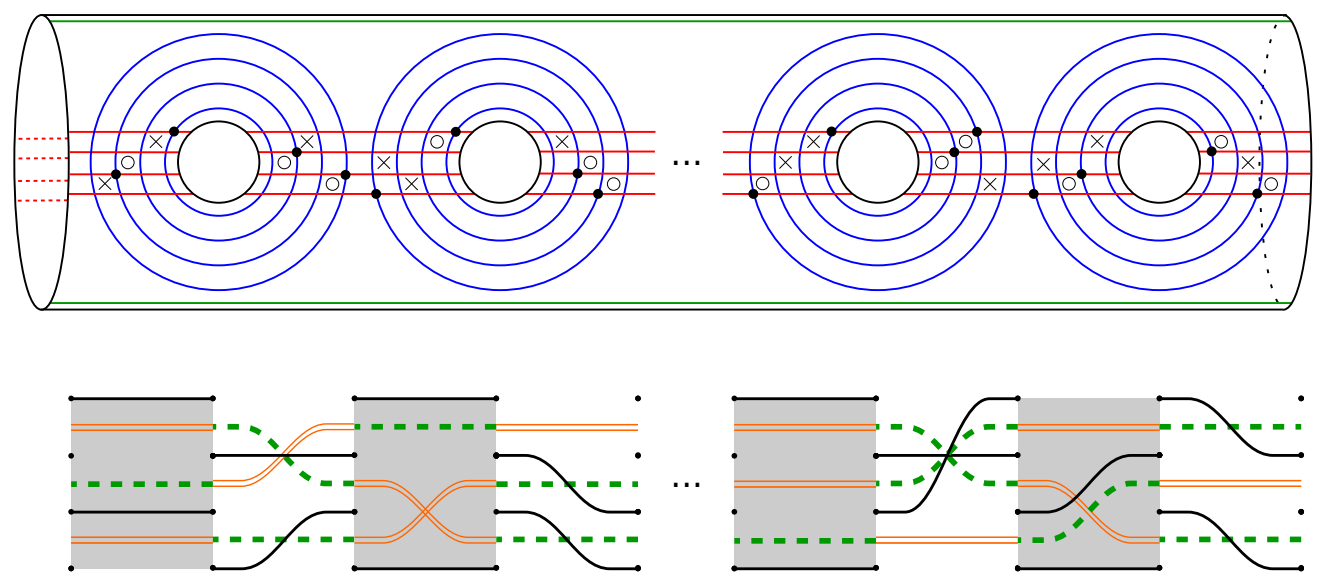

Figure 6: Top: a Heegaard diagram for $T$ coming from a plumbing of grids and the "bottommost" generator $\boldsymbol{x}_{\mathbb{O}}$. Bottom: the corresponding sequence of shadows for $T$, and the strands diagram for $\boldsymbol{x}_{\mathbb{O}}$.

corner $x_{4 i+3}^{\prime}$ of each grid $G_{4 i+3}$. Define $\boldsymbol{x}_{\mathbb{X}}^{\circ}$ analogously, by replacing $O_{j}$ with $X_{j}$ in the above definition.

Denote the $\beta$ circle containing each $x_{i}$ or $x_{i}^{\prime}$ by $\beta_{i}$ or $\beta_{i}^{\prime}$, respectively. Form a set of circles $\gamma$ by performing handleslides (which are allowed to cross $\mathbb{X}$ but not $\mathbb{O}$ ) of all $\beta_{i}$ and perturbations of all $\beta_{i}^{\prime}$, as in Figure 7. We look at the holomorphic triangle map associated to $(\Sigma, \boldsymbol{\alpha}, \boldsymbol{\beta}, \boldsymbol{\gamma}, \mathbb{O})$; see $[2 ; 6 ; 7]$. Let $k$ be the number of $O \mathrm{~s}$ in $\mathcal{H}$ (so the number of $O \mathrm{~s}$ in $\mathcal{H}^{\circ}$ is $k+2$ ). Observe that $(\Sigma, \boldsymbol{\beta}, \boldsymbol{\gamma}, \mathbb{O})$ is a diagram for $\left(\#^{k+2} S^{1} \times S^{2}\right)$, and let $\Theta$ be the top-dimensional generator. Let $\boldsymbol{y}$ be the generator of $(\Sigma, \boldsymbol{\alpha}, \boldsymbol{\gamma}, \mathbb{O})$ nearest to $\boldsymbol{x}_{\mathbb{O}}^{\circ}$. There is a holomorphic triangle that maps $\boldsymbol{x}_{\mathbb{O}}^{\circ} \otimes \Theta$ to $\boldsymbol{y}$, so $M\left(\boldsymbol{x}_{\mathbb{O}}^{\circ}\right)=M(\boldsymbol{y})$.

Observe that $(\Sigma, \boldsymbol{\alpha}, \boldsymbol{\gamma}, \mathbb{O})$ is a diagram for $S^{3}$ with $k+2$ basepoints, so, as a group graded by the Maslov grading, we have $\widehat{\operatorname{HF}}(\Sigma, \boldsymbol{\alpha}, \boldsymbol{\gamma}, \mathbb{O}) \cong H_{*+k+1}\left(T^{k+1}\right)$. The diagram has $2^{k+1}$ generators, so they are a basis for the homology. Let $\boldsymbol{y}^{\prime}$ be the generator obtained from $\boldsymbol{y}$ by replacing the intersection of $\gamma_{4 i+1}^{\prime}$ and the topmost $\alpha$ of $G_{4 i+1}$ with the intersection of $\gamma_{4 i+1}^{\prime}$ and the bottommost $\alpha$ of $G_{4 i+2}$, and the intersection of $\gamma_{4 i+3}^{\prime}$ and the bottommost $\alpha$ of $G_{4 i+3}$ with the intersection of $\gamma_{4 i+3}^{\prime}$ and the topmost $\alpha$ of $G_{4 i+4}$. There are $k$ disjoint bigons going into $\boldsymbol{y}^{\prime}$, so $M\left(\boldsymbol{y}^{\prime}\right) \leq-k$. The shaded $4 g$-gon on Figure 7 from $y^{\prime}$ to $y$ shows that $M\left(y^{\prime}\right)-M(y)=1$, so $M(y) \leq-k-1$. But the $2^{k+1}$ generators are a basis for the homology, so $M(y) \in$ $[-k-1,0]$. Thus, $M(y)=-k-1$, so $M\left(\boldsymbol{x}_{\mathbb{O}}^{\circ}\right)=-k-1$. We can also compute $M\left(x_{\mathbb{O}}\right)$ using the definition from [9, Section 3.4]. The computation is analogous to the one from $[9$, Section 6$]$, and we see that $M\left(\boldsymbol{x}_{\mathbb{O}}\right)=-k$. Note that $S\left(\boldsymbol{x}_{\mathbb{O}}^{\circ}\right)=a$, so 

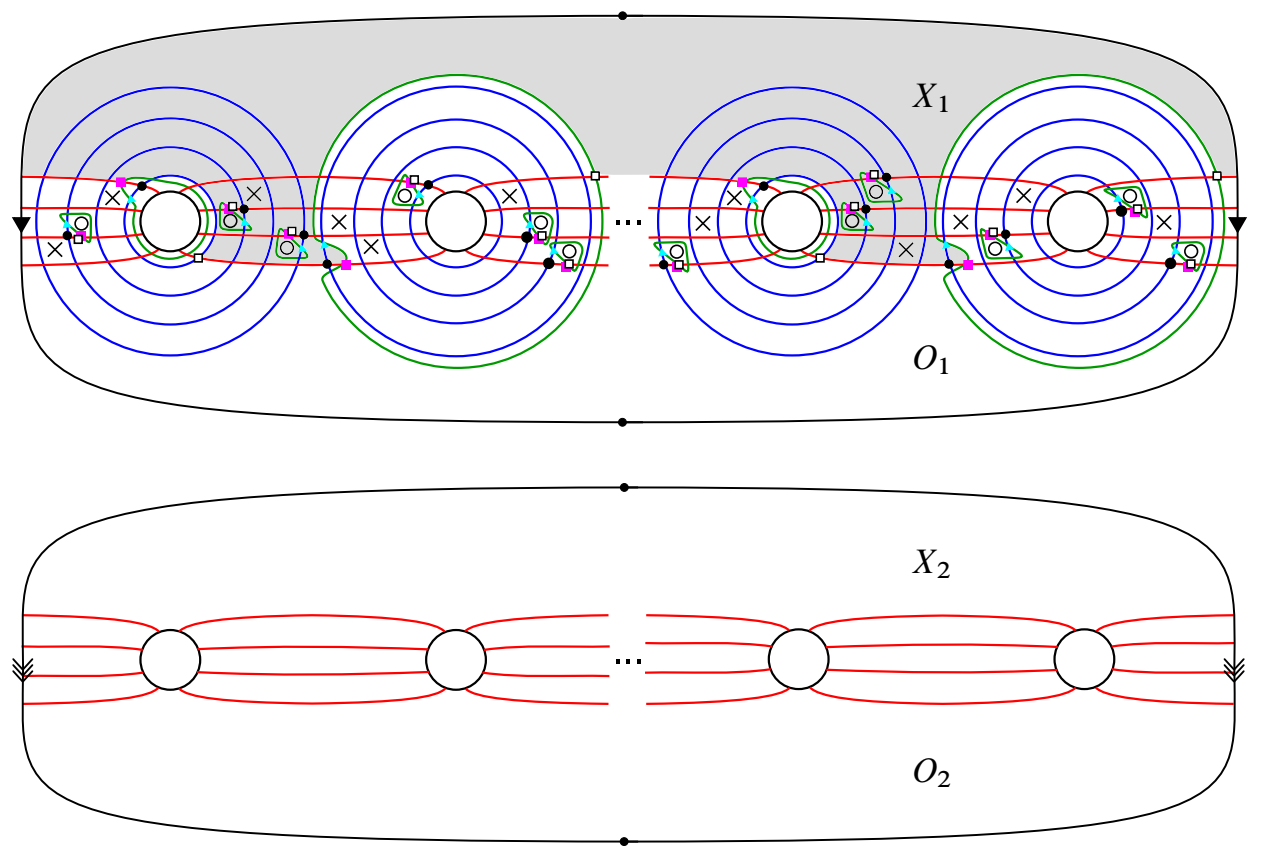

Figure 7: The Heegaard triple $(\Sigma, \boldsymbol{\alpha}, \boldsymbol{\beta}, \boldsymbol{\gamma}, \mathbb{O})$, with the original $\mathbb{X}$ markings left in. The black dots form the generator $\boldsymbol{x}_{\mathbb{O}}$, the purple squares form $\boldsymbol{y}$, the white squares from $\boldsymbol{y}^{\prime}$, and the cyan triangles form $\Theta$.

by Equation (1), for an arbitrary generator $\boldsymbol{x}_{i}^{\circ} \in \mathfrak{S}_{i}\left(\mathcal{H}^{\circ}\right)$ we have

$$
M\left(\boldsymbol{x}_{i}^{\circ}\right)-M\left(\boldsymbol{x}_{\mathbb{O}}^{\circ}\right)=M\left(\boldsymbol{x}_{i}\right)-M\left(\boldsymbol{x}_{\mathbb{O}}\right)+i-a,
$$

So

$$
M\left(x_{i}^{\circ}\right)=M\left(x_{i}\right)+i-a-1 .
$$

Similarly, for the $z$-normalized (or $\mathbb{X}$-normalized, to match the notation in this paper) grading $N$, we have $N\left(\boldsymbol{x}_{\mathbb{X}}^{\circ}\right)=-k-1$. Since $N=M-2 A-(k+2-l)$, we get

$$
A\left(\boldsymbol{x}_{\mathbb{X}}^{\circ}\right)=\frac{1}{2}\left(M\left(x_{\mathbb{X}}^{\circ}\right)-N\left(x_{\mathbb{X}}^{\circ}\right)-(k+2-l)\right)=\frac{1}{2}\left(M\left(x_{\mathbb{X}}^{\circ}\right)+l-1\right) .
$$

Again using the definition from [9, Section 3.4] as we do in [9, Section 6], we see that $M\left(\boldsymbol{x}_{\mathbb{O}}\right)=-k, N\left(\boldsymbol{x}_{\mathbb{X}}\right)=-k, A\left(\boldsymbol{x}_{\mathbb{X}}\right)=\frac{1}{2} M\left(\boldsymbol{x}_{\mathbb{X}}\right)$. Since $S\left(\boldsymbol{x}_{\mathbb{X}}\right)=n-a$, we have $M\left(x_{\mathbb{X}}^{\circ}\right)=M\left(x_{\mathbb{X}}\right)+n-2 a-1$, so we get

$$
A\left(\boldsymbol{x}_{\mathbb{X}}^{\circ}\right)=A\left(\boldsymbol{x}_{\mathbb{X}}\right)+\frac{1}{2}(n-2 a+l-2) .
$$

The Alexander multigrading on a generator $x^{\circ}$ can be described by the relative $\operatorname{Spin}^{c}$ structure $\mathfrak{s}\left(\boldsymbol{x}^{\circ}\right) \in \operatorname{Spin}^{c}\left(S^{3}, L\right)$; see [8]. In the case when the link is in $S^{3}$, one can think of $A_{i}$ by looking at the projection of a Seifert surface for $L_{i}$ onto $\mathcal{H}^{\circ}$. 
Specifically, for a generator $\boldsymbol{x}_{i}^{\circ} \in \mathfrak{S}_{i}\left(\mathcal{H}^{\circ}\right)$, we can compute its $A_{0}$ grading in the following way. Connect $X_{1}$ to $O_{1}$ and $X_{2}$ to $O_{2}$ away from $\boldsymbol{\beta}^{\circ}$, and $O_{1}$ to $X_{2}$ and $O_{2}$ to $X_{1}$ away from $\alpha^{\circ}$ to obtain a curve $C$ on $\mathcal{H}^{\circ}$ representing $L_{0}$, so that $C$ is negative the boundary of a disk $D$ that is a neighborhood of the rightmost grid (in general $C$ may be immersed but not necessarily embedded). Then

$$
A_{0}\left(\boldsymbol{x}_{i}^{\circ}\right)=\frac{1}{2}\left(e(D)+2 n_{\boldsymbol{x}_{i}^{\circ}}(D)-n_{\mathbb{X}}(D)-n_{\mathbb{O}}(D)\right)=i-\frac{1}{2}(n+1) .
$$

It follows that

$$
A^{\prime}\left(\boldsymbol{x}_{i}^{\circ}\right)=A\left(\boldsymbol{x}_{i}\right)-S\left(\boldsymbol{x}_{i}\right)+\frac{1}{2} l+n-a-1 .
$$

This completes the identification of gradings.

Remark The authors are in the process of upgrading the invariants in [9] to have Alexander multigradings, corresponding to different components of the tangle. The arguments in this paper automatically imply that the isomorphism from Theorem 3.2 respects the multigrading, with appropriate additive constants.

Last, we prove the Corollary to Main Theorem.

Proof of Corollary Fix $n$, and let $\mathbb{1}_{n}$ denote the trivial braid on $n$ strands (oriented from top to bottom). Let $B$ be an $n$-braid. Let $\mathcal{A}=\mathcal{A}\left(\partial^{1} \mathbb{1}_{n}\right)=\mathcal{A}\left(\partial^{1} B\right)$, and let $\mathrm{H}\left(\operatorname{Mod}_{\mathcal{A}}\right)$ be the homotopy category of right type $A$ modules over $\mathcal{A}$. Suppose that $-\otimes \widetilde{C T}(B)$ acts as the identity on $\mathrm{H}\left(\operatorname{Mod}_{\mathcal{A}}\right)$. Recall that one can recover the homotopy type of any $A A$ bimodule, ie $A_{\infty}$ bimodule, ${ }_{\mathcal{A}} N_{\mathcal{A}}$ from the functor $-\widetilde{\otimes} N$ on $\mathrm{H}\left(\operatorname{Mod}_{\mathcal{A}}\right)$, for example as $Q_{-\widetilde{\otimes} N}\left(\mathcal{A}_{\mathcal{A}}, \mathcal{A}_{\mathcal{A}}\right)$ in [10, (7.23) and (7.24)]. Thus, as $-\nabla \widetilde{C T}(B)=-\widetilde{\otimes}(\mathcal{A} \otimes \widetilde{C T}(B))$ is the identity, $\mathcal{A} \simeq \mathcal{A} \nabla \widetilde{C T}(B)$, so $\widetilde{C T}\left(\mathbb{1}_{n}\right) \simeq \widetilde{C T}(B)$. We show that the latter implies $B=\mathbb{1}_{n}$.

Let $k \geq 1$ be an integer such that $B^{k}$ is pure. Then

$$
\begin{aligned}
\widetilde{\mathrm{CT}}\left(B^{k}\right) & \simeq \widetilde{\mathrm{CT}}(B) \otimes \cdots \nabla \widetilde{\mathrm{CT}}(B) \\
& \simeq \widetilde{\mathrm{CT}}\left(\mathbb{1}_{n}\right) \otimes \cdots \nabla \widetilde{\mathrm{CT}}\left(\mathbb{1}_{n}\right) \\
& \simeq \widetilde{\mathrm{CT}}\left(\mathbb{1}_{n}^{k}\right) \simeq \widetilde{\mathrm{CT}}\left(\mathbb{1}_{n}\right) .
\end{aligned}
$$

Taking Hochschild homology, it follows that $\widehat{\operatorname{HFL}}\left(\left(B^{k}\right)_{0}, 0\right) \cong \widehat{\operatorname{HFL}}\left(\left(\mathbb{1}_{n}\right)_{0}, 0\right)$. Recall that, in our notation, [1, Theorem 1(b)] says that if there is a triply graded isomorphism $\widehat{\mathrm{HFL}}\left(T_{0}, 0\right) \cong \widehat{\mathrm{HFL}}\left(\left(\mathbb{1}_{n}\right)_{0}, 0\right)$ and $T$ is a pure braid, then $T=\mathbb{1}_{n}$. Thus, $B^{k}=\mathbb{1}_{n}$. Since the braid group is torsion-free, it follows that $B=\mathbb{1}_{n}$. 


\section{References}

[1] J A Baldwin, J E Grigsby, Categorified invariants and the braid group, Proc. Amer. Math. Soc. 143 (2015) 2801-2814 MR3336606

[2] R Lipshitz, C Manolescu, J Wang, Combinatorial cobordism maps in hat HeegaardFloer theory, Duke Math. J. 145 (2008) 207-247 MR2449946

[3] R Lipshitz, PS Ozsváth, DP Thurston, Bimodules in bordered Heegaard-Floer homology, Geom. Topol. 19 (2015) 525-724 MR3336273

[4] C Manolescu, An introduction to knot Floer homology, preprint (2016) arXiv: $1401.7107 \mathrm{v} 2$

[5] C Manolescu, P Ozsváth, Z Szabó, D Thurston, On combinatorial link Floer homology, Geom. Topol. 11 (2007) 2339-2412 MR2372850

[6] P Ozsváth, Z Szabó, Holomorphic disks and three-manifold invariants: properties and applications, Ann. of Math. 159 (2004) 1159-1245 MR2113020

[7] P Ozsváth, Z Szabó, Holomorphic triangles and invariants for smooth four-manifolds, Adv. Math. 202 (2006) 326-400 MR2222356

[8] P Ozsváth, Z Szabó, Holomorphic disks, link invariants and the multi-variable Alexander polynomial, Algebr. Geom. Topol. 8 (2008) 615-692 MR2443092

[9] I Petkova, V Vértesi, Combinatorial tangle Floer homology, preprint (2015) arXiv: $1410.2161 \mathrm{v} 2$

[10] P Seidel, Lecture notes on categorical dynamics and symplectic topology (2013) Available at http://www-math.mit.edu/ seidel/937/lecture-notes.pdf

Department of Mathematics, Columbia University

Room 509, 2990 Broadway, New York, NY 10027, United States

Institut de Recherche Mathématique Avancée, Université de Strasbourg

7 rue René Decartes, 67084 Strasbourg, France

ina@math.columbia.edu, vertesi@math.unistra.fr

http://math.columbia.edu/ ina, http://www-irma.u-strasbg.fr/ vertesi/

Received: 4 April 2015 Revised: 19 November 2015 
